\title{
Alpha-glucosidase inhibitor
}

National Diabetes Information Clearinghouse (NDIC)

\section{Definitions}

Type 2 diabetes

Defined by National Diabetes Information Clearinghouse (NDIC)

Carbohydrate

Defined by National Diabetes Information Clearinghouse (NDIC)

\section{Blood glucose}

Defined by National Diabetes Information Clearinghouse (NDIC)

\section{Source}

National Diabetes Information Clearinghouse (U.S.). (2009). The diabetes dictionary. [Bethesda, Md.]: U.S. Dept. of Health and Human Services, National Institutes of Health, National Institute of Diabetes and Digestive and Kidney Diseases, National Diabetes Information Clearinghouse.

A class of oral medicine for type 2 diabetes that slows down the digestion of foods high in carbohydrate, such as rice, bread, milk, and fruit. The result is a slower and lower rise in blood glucose after meals. (Generic names/Brand names: acarbose/Precose; miglitol/Glyset.) 